CONCURSO ESTUdiANTIL DE DISEÑO

\title{
Diseño de una Herramienta para Entregar en el Lugar aPoyo al indigente: HELPi
}

\author{
Alain Díaz-Montes, Carlos G. Verdín, Carlos A. \\ Puga, Ricardo Rodríguez-Torres, Marcela D. \\ Rodríguez
}

Publicado: 31 Octubre 2018

\begin{abstract}
Resumen
Dado los altos índices de indigencia que caracterizan a nuestro país, Mexicali destaca por la indigencia debida al fenómeno de migración. Para entender el problema realizamos entrevistas a indigentes y coordinadores de asociaciones que ofrecen apoyo en la comunidad. Lo anterior ayudó a identificar requerimientos que derivaron en una pulsera digital que permite notificar de los servicios de apoyo disponibles en la comunidad. Presentamos un diseño de concepto y un escenario de uso para ilustrar su funcionalidad y utilidad del sistema. Una presentación del concepto del diseño con la Directora del Desarrollo Integral para la Familia (DIF) del municipio, nos permitió confirmar su potencial utilidad y adopción.
\end{abstract}

Palabras clave: Indigente; Dispositivo Vestible; Diseño de Escenarios.

\section{Introducción}

La indigencia se clasifica como pobreza, falta de medios para vivir, falta de medios para alimentarse, vestirse. Según la CEPAL en sus estadísticas el porcentaje de personas indigentes en América Latina en 2012 fue de $11.3 \%$ lo que equivale a 66 millones de personas [1].

En México el porcentaje de pobreza extrema durante el 2016 fue de $7.6 \%$ lo que equivale a 8.6 millones de personas. A nivel entidad, el porcentaje de personas en situación de pobreza extrema en B.C. es de $1.1 \%$ lo que equivale a 39 mil personas [2]. Sin embargo se desconoce una estimación precisa de la cantidad de indigentes. En comparación con Estado Unidos que se estima que 553,742 habitantes son indigentes [3]. Localmente, aunque encuestas oficiales realizadas en Mexicali B.C. por INEGI indican que alrededor de 300 personas viven en condición de calle, la realidad es que cada vez son más los indigentes que deambulan por las calles y van en aumento. Son muchas más que las 300 que

Díaz-Montes, A. Verdín, CG., Puga, CA., Rodríguez-Torres, R., Rodríguez, MD.

Universidad Autónoma de Baja California. Facultad de Ingeniería

Mexicali.

Mexicali, Baja California.

Email: adiaz56@uabc.edu.mx,verdinc@uabc.edu.mx,

carlos.puga@uabc.edu.mx,jesus.ricardo.rodriguez.torres@uabc.edu.mx, marcerod@uabc.edu.mx señala el INEGI y es difícil estimar una cifra exacta [4]. Esto se debe a que, Mexicali y Tijuana enfrentan problema de migración ya que son ciudades fronterizas a E.U. Todos los días llegan migrantes a Mexicali, que intentan cruzar ilegalmente hacia E.U, y que al fallar, permanecen aquí como indigentes. Lo anterior, aunado a que el calor extremo que caracteriza a la ciudad de Mexicali, obliga al gobierno a aplicar programas de apoyos especiales para proteger a estas personas. En este artículo, presentamos el diseño de un concepto de un dispositivo vestible para facilitar a diversas organizaciones monitorear y proveer apoyos a los indigentes-migrantes que deambulan por la ciudad.

\section{Trabajo Relacionado}

Para comprender las limitaciones e implicaciones para diseñar artefactos digitales que mejoren las condiciones del indigente joven, en [5] se presenta un estudio basado en entrevistas contextuales. De este se concluye que los indigentes tienen restricciones para acceder a infraestructura de internet y electricidad para recargar la batería de sus dispositivos. Para ellos, el traspasar ciertos lugares considerados públicos, tal como librerías, podría tener severas consecuencias.

Similarmente, se han realizado encuestas para conocer la penetración de los teléfonos móviles en la comunidad indigente. En San José, California, se identificó que el 68\% de los indigentes tienen teléfono celular. Sin embargo, sólo el $50 \%$ tiene acceso a internet desde sus teléfonos. Uno de los aspectos que se identificaron, es la dificultad de mantener informados a los indigentes de los servicios disponibles en el área dada su dispersión, tal como eventos que se programan para dar servicios de atención a la salud. Con base a lo anterior, se propuso desarrollar una plataforma-web que permitiera a las organizaciones disponibles en el área, enviando mensajes SMS para anunciar sus servicios de asistencia a los indigentes con teléfono celular; evitando así, la necesidad de contar con acceso a internet [6]. Similarmente, en [7] se propone tecnología vestible basada en códigos QR (Quick Response), los cuales se usan para configurar los teléfonos celulares de indigentes para que reciban diferentes tipos de notificaciones sobre servicios proporcionados por las diferentes agencias, tal como horarios de apertura o notificaciones personalizadas para recordarles tomar su medicamento. La ventaja de separar las funcionalidades de configuración de los recordatorios (mediante los QR), de la aplicación móvil que los muestra, es que si los indigentes cambian 
o pierden su teléfono, los QR le permitirán configurar un nuevo teléfono.

Los trabajos anteriores, limitan la adopción de las aplicaciones que proponen aplicaciones que sólo pueden ser utilizados por indigentes que cuenten con teléfono celular, lo cual en nuestro contexto no siempre es posible. Se ha estudiado que sólo una proporción pequeña de migrantes que llegan a los centros de apoyo cuentan con teléfonos [8].

\section{Metodología}

Siguiendo el enfoque de Diseño Centrado en el Usuario [9], se realizó una iteración de esta metodología, los cuales incluyeron las siguientes etapas:

\subsection{Caso de Estudio}

Se identificaron mediante entrevistas semi-estructuradas las diferentes necesidades que tiene la población indigente-migrante. Todos los autores de este artículo, contactamos en parques públicos a individuos que presentan esta situación, para obtener información sobre: propósito de migrar, apoyos que recibe, comunicación con sus familiares, socialización local, y sus necesidades, entre otras. Mediante un análisis temático simple, analizamos los datos obtenidos [10]. Esto consistió en 2 sesiones de interpretación, en las cuales los autores analizamos las respuestas de las entrevistas e identificamos temas.

\subsection{Diseño Basado en Escenarios}

Se utilizó el diseño basado en escenarios, cuyo principal propósito es proveer una "descripción de personas utilizando tecnología" lo que nos ayudó a discutir y analizar cómo la tecnología está (o estará) afectando sus actividades [9]. Como resultado, realizamos un video representativo del problema de indigencia, así como la solución planteada por el equipo, con la finalidad de ser expuesta ante autoridades encargadas de esta problemática en el municipio de Mexicali.

\section{Resultados del Caso de Estudio}

Tal como se presenta en la Tabla 1, de los 6 migrantes-indigentes que participaron en el estudio, la mitad reportó usar celulares para comunicarse con sus familias o redes sociales; solamente dos participantes tenían familiares en la ciudad y todos provenían de diferentes estados del país, excepto 1 quien reportó haber sido deportado de E.U. Todos los participantes eran migrantes masculinos, aunque esto no quiere decir que no existan mujeres indigentes, lo cual nos lo confirmó la Directora del DIF durante la sesión de evaluación quien comentó que la mayoría de los migrantes son hombres.

Tabla 1. Descripción de participantes

\begin{tabular}{cccccc}
\hline Sujeto & Procedencia & $\begin{array}{c}\text { Tiempo en } \\
\text { Mexicali }\end{array}$ & $\begin{array}{c}\text { Familiares } \\
\text { en Mexicali }\end{array}$ & Género & $\begin{array}{c}\text { Uso de } \\
\text { Tecnologias }\end{array}$ \\
\hline S1 & Morelia & 10 meses & Si & Masculino & No \\
S2 & Michoacán & Varios dias & No & Masculino & No \\
S3 & Sinaloa & $3-5$ años & Si & Masculino & Si (celular) \\
S4 & Oaxaca & 3 meses & No & Masculino & No \\
S5 & $\begin{array}{c}\text { Jalisco } \\
\text { (migrante) }\end{array}$ & 2 meses & No & Masculino & $\begin{array}{c}\text { Si (celular y } \\
\text { Facebook) }\end{array}$ \\
S6 & $\begin{array}{c}\text { Migrante } \\
\text { deportado }\end{array}$ & 3 años & No & Masculino & $\begin{array}{c}\text { Si } \\
\text { (Facebook) }\end{array}$ \\
\hline
\end{tabular}

4.1 Temas principales de las entrevistas

Analizando los transcriptos de las entrevistas se encontró que:

\subsubsection{Los indigentes buscan oportunidades de trabajo.}

El $50 \%$ de los participantes concordaron que tienen problemas para conseguir trabajo debido a la falta de identificación, en particular el sujeto 6 nos dijo: "Necesito dinero para irme a Estados Unidos, trabajo 3 o 4 días por semana, siempre ando consiguiendo trabajo pero no tengo documentos oficiales" [S6].

\subsubsection{Los programas de apoyo son poco flexibles}

Identificamos que 3 participantes se quejaron de los servicios que ofrecen los centros y albergues: El sujeto 5 nos comentó: "Un día fui a las 8:00 pm a solicitar comida a [uno de los centros], porque a esa hora se supone dan la comida, pero ya no me quisieron atender...”. El sujeto 6 nos dijo: “Al principio [cuando llegó a Mexicali] fui a un centro, pero ahí sólo te atienden por 3 días, te dan de comer, y te puedes bañar y dormir". Además de las inconformidades de los participantes hacia estos centros de ayuda, el sujeto 2 nos comentó que ni siquiera conocía estos albergues, y que desearía obtener información acerca de estos apoyos ya que nos preguntó: "ustedes tienen un folleto de información".

\subsubsection{Existe empatía entre los indigentes}

El sujeto 1 nos dijo que tiene un círculo de amistades en los lugares que frecuentan, pero al mismo tiempo existe desconfianza al momento de conocer a otras personas indigentes, ya que han sufrido de asaltos. Y el sujeto 3 dijo: "Cuando tengo problemas con mi esposa, me vengo al parque, aqui si me entienden”.

\subsection{Requerimientos a abordar}

Dado las respuestas que obtuvimos del caso de estudio se concluyó que los requerimientos del sistema son:

a. Bajo costo. Aunque este es un requerimiento importante para que pueda ser viable su adopción en un contexto real; lo que nos motiva a proponer este requerimiento es evitar despertar el interés del indigente por obtener una remuneración económica intentando venderlo.

b. Interacción implícita. El sistema debe de ser fácil de entender y usar. Consideramos que su funcionalidad será como visualizador.

c. De propósito específico. El sistema debe consistir en componentes especializados que ofrecen un conjunto de funciones específicas para limitar la posibilidad en que pueda ser utilizado para otros fines.

d. Notificaciones oportunas. El sistema debe hacer consciente a los indigentes-migrantes sobre los apoyos disponibles, de forma oportuna. Tal como, entrega de comida, campañas de salud u oportunidades de trabajo.

e. Personalizable. El sistema debe facilitar a las asociaciones comunicar a los indigentes los apoyos particulares que ofrecen; pero también debe permitir enviar notificaciones personalizadas a las necesidades de los indigentes, por ejemplo, enviar mensajes sobre una oportunidad de trabajar como ayudante de albañil.

\section{Diseño}

Para abordar los requerimientos anteriores, se diseñó HELPi (Herramienta para Entregar en el Lugar aPoyo al indigente). Este consiste de un dispositivo vestible que entrega notificaciones sobre apoyos que ofrecen los centros de desarrollo social. Está formado por una Pulsera Digital y un Sistema Administrador basado-Web. 


\subsection{Pulsera Digital}

Este consta de tres elementos que definen su interacción (ver Figura 1):

Pines digitales. Estos permitirán la personalización de las notificaciones. Identificamos 4 tipos básicos de servicios que ofrecen los centros de apoyo: Salud, Alimentación, Centro de Albergue e Hidratación. Por lo que los centros enviarán mensajes personalizados con base a estas categorías (Ver Figura 2 y requerimiento 3 y 5).

Pantalla de despliegue: Muestra mensajes simples personalizados, indicando el apoyo específico a ofrecer, el lugar y momento que se ofrecerá (ver requerimiento 2).

Tecnología RFID (del inglés Radio Frequency Identification): Permite personalizar la pulsera digital a cada indigente. Adicionalmente, ayudaría a monitorear y llevar un control sobre el indigente, tal como apoyos que requiere, apoyos recibidos, y mantener su información demográfica.

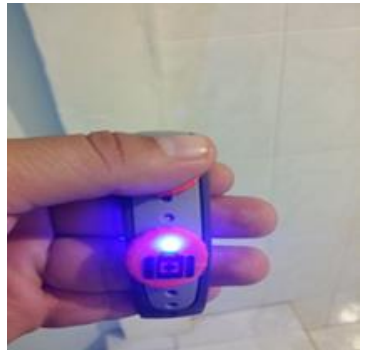

a)

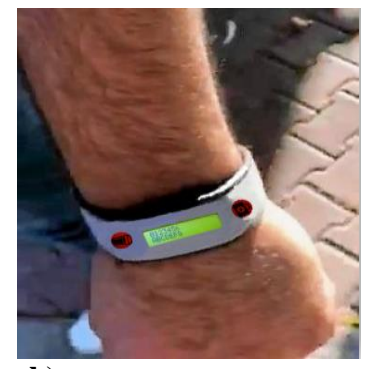

b)
Figura. 1. Pulsera Digital con: a) PIN recibiendo una notificación y b) Pantalla monocromática para recibir mensajes

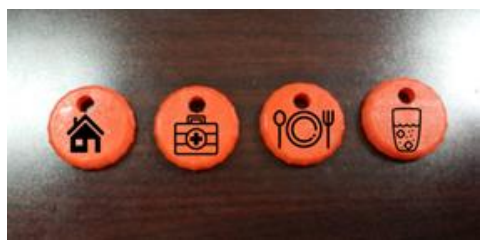

Figura 2. Ejemplos del PIN Digital

\subsection{Administrador Basado en el Web}

HELPi cuenta con un sistema web que permite el registro de personas que reciban la pulsera digital, así como configurar la entrega de las notificaciones. El subsistema de Registro, permite almacenar los datos generales del indigente, así como datos sobre su procedencia como la fecha de llegada (ver figura 3). Adicional a esto, se podrá configurar el tipo de notificación que se desea enviar a los afiliados (ver figura 4), donde se introducirán los datos del apoyo a ofrecer, como lugar, fecha, hora, y una opción para repetir el envío de la notificación para recordar a las personas afiliadas de la entrega del apoyo nuevamente (requerimiento 4).

\subsection{Escenario de Uso}

Para describir el diseño de interacción de HELPi, se presenta el siguiente escenario:

En el Centro de Desarrollo Integral Familiar (DIF) se encuentra Luis, el asistente que administrar apoyos a personas indigentes. Luis recibe al migrante Juan, y le solicita sus datos para almacenarlos en la base de datos de la aplicación (Figura 3). Después le entrega la Pulsera Digital, y le explica cómo funciona. Luis le dice a Juan que la pulsera le enviará notificaciones por medio de sus pines digitales los cuales se enfatizan activando una luz; y que mediante el mensaje de texto que se visualizará en la pantalla podrá saber la ubicación y la hora de su entrega. Dadas las altas temperaturas de Mexicali, el DIF entregará agua y electrolitos a personas de bajos recursos, por lo que Luis decide enviar una notificación a los migrantes. Entra a la aplicación (ver Figura 4) para dar de alta una notificación e indica que ésta se repartirá en el parque El Cachanilla del 11 al 15 de julio, de 4:00-6:00 pm. Encontrándose Juan, en ese momento en el centro de la ciudad, recibe la notificación visualizando que el pin de ayuda alimenticia (Figura 1a) está encendido, y lee en la pantalla (Figura 1b) que la entrega será en uno de los parques cercanos a su ubicación ese mismo día, por lo que se dispone a recoger su despensa. Juan llega al parque y el grupo de apoyo DIF le solicita que muestre su pulsera. Luis acerca un dispositivo móvil a la pulsera de Juan para escanear su código RFID, lo que permite registrar que recibió el apoyo.

5.4 Implementación del diseño.

Se considera implementar una versión semi-funcional del prototipo para evaluarse en el contexto real. En la Tabla 2 se presentan los costos estimados de implementar un prototipo con los componentes electrónicos indicados.

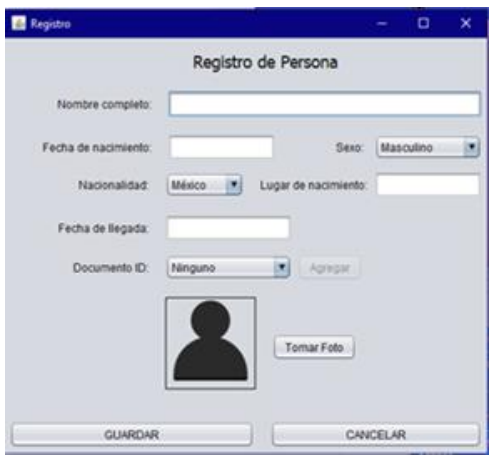

Fig. 3. Ventana de Registro de Persona

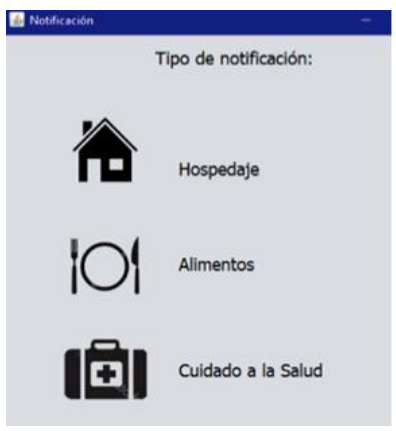

a)

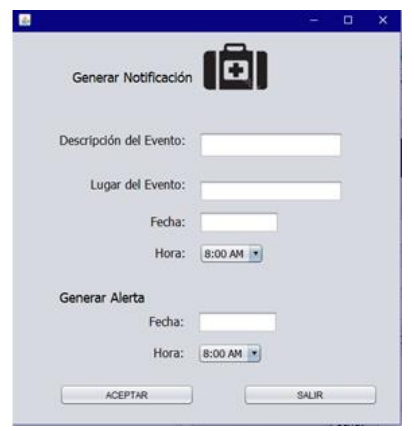

b)
Figura 4. Interfaces de usuario para: a) acceder a la configuración de las notificaciones y b) datos del apoyo.

\section{Evaluación del Concepto}

Con el propósito de conocer la percepción de la utilidad y la factibilidad de su uso por personas que dirigen los programas de apoyo social al indigente, se realizó una presentación a la Directora de los programas del DIF del Municipio de Mexicali y el Coordinador General de Formación Profesional y Vinculación Universitaria (Ver Figura 5). Esta consistió en una sesión de 1 hora y media aproximadamente, realizada en uno de los centros del DIF. Se presentó el propósito de la disciplina del estudio en la que se propone contribuir (IHC), el contexto del problema social 
de indigencia, el concepto del diseño propuesto y finalmente, se discutió la factibilidad de usar el dispositivo. Para este propósito, planteamos preguntas, tales como ¿Cuál es su opinión respecto sobre la utilidad de la pulsera digital? ¿Cuáles funciones son adecuadas o inadecuadas para abordar las necesidades de los migrantes? ¿Qué otros usos podría tener la pulsera? Durante la sesión se tomaron notas sobre los comentarios dados, para su posterior análisis temático [10].

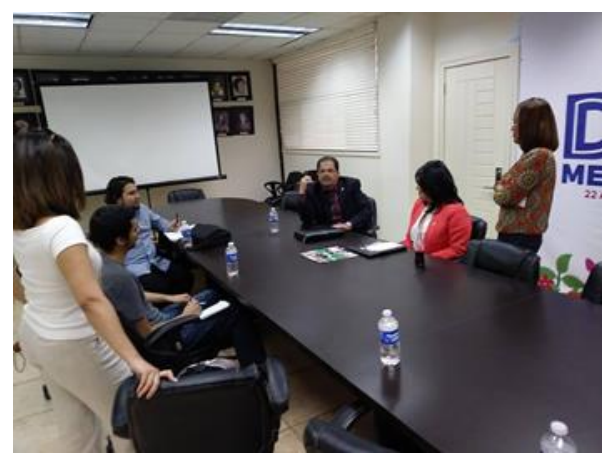

Figura 5. Reunión con la Presidenta del DIF.

Tabla 2. Costos Estimados

\begin{tabular}{ccc}
\hline Nombre & Descripción & Costos \\
\hline $\begin{array}{c}\text { Módulo de Lora RF } \\
\text { Arduino }\end{array}$ & Radiotransmisor & 4 dlls \\
\hline $\begin{array}{c}\text { Pantalla } \\
\text { Monocromática }\end{array}$ & $\begin{array}{c}\text { Es una placa electrónica } \\
\text { donde viene montado un } \\
\text { microcontrolador }\end{array}$ & 2 dlls \\
\hline Circuito Impreso & $\begin{array}{c}\text { Circuito que va integrado en } \\
\text { solo color. }\end{array}$ & 2 dispositivo \\
\hline Bateria LiPo & $\begin{array}{c}\text { Batería recargables que } \\
\text { suelen utilizar los sistemas } \\
\text { eléctricos de radiocontrol } \\
\text { Pulsera RFID }\end{array}$ & $\begin{array}{c}\text { RFID (identificación por } \\
\text { radiofrecuencia) frecuencia } \\
\text { permiten leer el código a una } \\
\text { distancia cercana }\end{array}$ \\
\hline
\end{tabular}

\subsection{Resultados}

De la evaluación al diseño, obtuvimos retroalimentación que nos ayudó a identificar la percepción de utilidad e interés en adoptarlo. La Presidenta del DIF confirmó que le parecía adecuado el sistema. Proporcionó información acerca del problema a abordar, por ejemplo, dijo: "Actualmente, no hay forma segura de saber [refiriéndose a los indigentes] si hay comida, refugio o servicios de salud", y comentó, que el albergue del DIF abre sólo en ciertas temporadas del año; y que ofrece alimentación en ciertos parques de la ciudad. Lo anterior porque otras organizaciones cubren otras necesidades. Estos comentarios sugieren que un sistema como el que proponemos ayudaría a dar a conocer la disponibilidad de los servicios que ofrecen las diversas asociaciones del municipio, los cuales varían en temporadas del año. Adicionalmente, cuestionaron aspectos que podrían impactar su adopción. En particular, realizaron preguntas que nos demostraron interés en conocer la funcionalidad del sistema para que fuera utilizada en el contexto real. Por ejemplo, preguntaron sobre el costo (ver Tabla 2), y quien lo administraría. Y mostraron preocupación sobre aspectos de seguridad, tal como riesgo de robar la pulsera al indigente y que pudiera darse mal uso a la información del indigente asociada a la pulsera.

\section{Conclusiones y Trabajo Futuro}

Consideramos que el diseño propuesto ayudaría a mejorar el acceso a los servicios que se ofrecen en la comunidad, mediante nuevas tecnologías intuitivas para los usuarios. Se planea extender la evaluación al diseño del conceptual, mejorar el prototipo e implementarlo para realizar una evaluación de usabilidad en el contexto real. Consideramos que uno de los retos que se aborda es proporcionar una tecnología ayude a comunicar servicios de apoyo a una comunidad indigente-migrante, la cual se caracteriza por estar dispersa en la ciudad.

\section{Agradecimientos}

A la Directora del (DIF) y a los participantes del estudio.

\section{Referencias}

[1] Inclusive Social Development: he next generation of policies for overcoming poverty and reducing inequality in Latin America and the Caribbean. Cepal Report. Disponible en: https://www.cepal.org/en (accessed on 17th June 2018)

[2] https://www.coneval.org.mx

[3] 'The State of Homelessness in America', https://endhomelessness.org/homelessness-inamerica/homelessness-statistics/state-of-homelessnessreport/

[4] http://www.beta.inegi.org.mx/

[5] Woelfer, J.P., Hendry, D.G. Homeless young people and living with personal digital artifacts. CHI '11, ACM, NY, 1697-1706.

[6] Figueira, S., Linnell, N. and Fong, N. "StreetConnect: SMS announcements for homeless people," 2013 IEEE Global Humanitarian Tech. Conf. (GHTC), CA, 2013, 495-500.

[7] Gebrekristos, M., Aljadaan, A., Bihani, K. QR-Codes for the chronically homeless. CHI EA'08. ACM, NY, 3879-3884.

[8] Newell, B.C., Gomez, R., and Guajardo, V,. E. Information seeking, technology use, and vulnerability among migrants at the United States-Mexico border, The Information Society, 32:3, (2016), 176-191

[9] Rosson, M.B., and Carroll, J.M. 2002. Usability Engineering, San Francisco, CA: MK Publishers.

[10] Braun, V., and Clarke, V., Using thematic analysis in psychology. Qualitative Research in Psychology 2006; 3:77101 